\title{
Influence of Kinesio tape on lower limb muscular activity after knee joint rehabilitation program
}

\begin{abstract}
This study aimed to identify the effect of using Kinesio tape on the neuromuscular activity of quadriceps muscle after the rehabilitation program of the knee joint. Ten players participated in present study (age: $26.20 \pm 1.69$ years; body mass: $77.10 \pm 4.48$ $\mathrm{kg}$; height: $172.30 \pm 5.46 \mathrm{~cm}$ ). The EMG signals were stored at a sampling frequency of $1000 \mathrm{~Hz}$ and digitized using a 16-bit analogue to digital (A/D) converter. EMG data were processed using ProEMG software (Myon 320, Schwarzenberg, Switzerland). Raw EMG data was band-pass $(20 \mathrm{~Hz}-450 \mathrm{~Hz}$ ) applying a Butterworth filter. The signals were preprocessed using full-wave rectified and a linear envelope obtained using the Root Mean Square (RMS) approach with a window size of 300 ms. Data was normalised to the peak muscle activity value over each attempt (\%MAX). Descriptive statistics were reported as means and standard deviations (mean \pm Std. Deviation). The normality of the data was analyzed using the Shapiro-Wilk test. Independent Ttest was used to detect significant differences and compare the mean of each variable between single-leg squat and balance tests. The statistical analysis was performed using IBM SPSS software Statistics v21 SPSS Inc., USA) with significance level defined as $p<0.05$ for each test. The results of the present study showed that applying KT around the knee joint after rehabilitation program does not promote immediate changes or effects on the lower limb muscle activities of (VL, RF, VM, BF, ST, L_GAST, and M_GAST
\end{abstract}

Keywords: Kinesio tape; Injury-prevention; Electromyography; Knee joint. 


\section{Introduction}

In the last decade, Kinesio taping became the most commonly used tape in the field of sport where it was used in the treatment and rehabilitation of professional players (Huang, Hsieh, Lu, \& Su, 2011; Serrão et al., 2016), in the international championship and world cup. Some studies showed that Kinesio taping had a lot of positive effects on neuromuscular activity and at the same time, it doesn't restrict joint mobility but still, there is lack of objectivity and need many studies (Chang, Chen, Lee, Lin, \& Lai, 2015; Wageck, Nunes, Bohlen, Santos, \& de Noronha, 2016).

Kinesio taping is considered one of the most common and recent used techniques, where it is used as a therapeutic technique for pain relief (Aghapour, Kamali, \& Sinaei, 2017; Chang et al., 2015; Pires et al., 2019), correction of joint position (Wageck et al., 2016), prevention of oedema and injuries, improve range of motion and muscular activity for the individual. In addition to this type of taping has a lot of positive effects, where due to its elasticity (Chang et al., 2015; Kocyigit et al., 2015), skin can be folded and allow more space under the skin that allows good circulation to the injured area and improve performance and strengthening muscles and joints (Chang et al., 2015).

Chang et al. (2015) and Hebert-Losier, Yin, Beaven, Tee, and Richards (2019) reported that Kinesio tape stimulates circulation, relieve pain, improve efficiency, performance and tone of muscles and also stimulate the lymphatic system. Consequently, it decreases the incidence of inflammation and oedema or swelling. Nwe, Tun, Aung, and Myaing (2019) demonstrated that when using Kinesio tape on knee joint with performing some traditional exercises in individuals with osteoarthritis, this led to positive results where it relieved pain, decreased stiffness of knee joint and led to improving the performance of knee joint compared to not using Kinesio tape .

Doğan et al. (2019) showed that when using Kinesio tape led to positive effect and improvement of flexion and extension of knee and increase in neuromuscular activity of knee. Juchler, Blasimann, Baur, and Radlinger (2016) reported that electromyography (sEMG) is considered one and the common of instruments that are used for identifying and measuring the neuromuscular activity of muscle through studying characteristics of the neuromuscular system, where during rest, the muscle 
produces very low electrical activity which is known as (muscle tone) while when the muscle is activated (stimulated).

The use of anatomical analysis of the muscular system is considered one of the scientific methods for studying communicating areas of muscles with joints. It is also used by the coach to identify the most important muscles that affect positively on mobility to promote the functional performance of muscles in an appropriate way. Despite, the studies of Doğan et al. (2019) and Nwe et al. (2019) indicated that Kinesio tape had positive effects when it is used on ankle joint and knee joint, several studies on the literature, reported that a lack of significance of the effects of Kinesio tape on muscle activity and muscle strength (Aghapour et al., 2017; Fu et al., 2008; Poon et al., 2015; Wong, Cheung, \& Li, 2012).

Thus, this study aims to identify the effect of using Kinesio tape on the neuromuscular activity of quadriceps muscle after the rehabilitation program of the knee joint. This will contribute to a decreasing period for the program in an appropriate way.

\section{Material and Methods}

\section{Participants}

Ten players participated in present study (age: $26.20 \pm 1.69$ years; body mass: $77.10 \pm$ $4.48 \mathrm{~kg}$; height: $172.30 \pm 5.46 \mathrm{~cm}$ ). The players' consent was obtained, and the study was approved by the institutional ethics committee of studies and research.

\section{Experiment Protocol}

Before the data collection, all participants were informed about the study processes. All participants executed two tests (single-leg squat and balance test), by with and without Kinesio taping. A $5 \mathrm{~cm}$ width of Kinesio tape (Tmax Tape, Tmax Medical Co., Ltd, Goyang, Gyeonggi, South Korea) was cut into a Y shaped Kinesio tape, applied to the patella, wrapping the patella medially and laterally with $25 \%$ tension, according to the recommendation (Castrogiovanni et al., 2016). 
After the brief warm-up and practice, participants performed the single-leg test according to Hall, Paik, Ware, Mohr, and Limpisvasti (2015), then balance test in the following sequence: first, the subject performed five trials of a single-leg squat test without tape as the baseline trials with 5 minutes interval resting; second, the elastic tapes were applied around the unilateral knee joint by an experienced physical rehabilitation specialist; third, to let participants obtain full resting and avoid muscle fatigue induced by the previous trials, after 15 minutes with the tape, and repeat the three sequences in balance test. Upon arriving for a test session, the electrical muscle activity of the lower injured limb muscles, the vastus lateralis (VL), rectus femoris (RF), vastus medialis (VM), biceps femoris (BF), semitendinosus (ST), lateral gastrocnemius (L_GAST), and medial gastrocnemius (M_GAST) were recorded using surface EMG system (Myon m320RX; Myon, Switzerland). The skin over the muscles of the dominant leg was shaved and cleaned with alcohol And bipolar, circular $10 \mathrm{~mm}$ diameter silver chloride surface electrodes (SKINTACT FS-50, Robohardware kft, Hungary) were secured on the selected muscles. Electrodes were attached over each muscle following the SENIAM guidelines maintaining a $2 \mathrm{~cm}$ centre to centre interelectrode spacing (Hermens, Freriks, Disselhorst-Klug, \& Rau, 2000).

\section{Data analysis}

The EMG signals were stored at a sampling frequency of $1000 \mathrm{~Hz}$ and digitized using a 16-bit analogue to digital (A/D) converter. EMG data were processed using ProEMG software (Myon 320, Schwarzenberg, Switzerland). Raw EMG data was band-pass (20 $\mathrm{Hz}-450 \mathrm{~Hz}$ ) applying a Butterworth filter. The signals were preprocessed using fullwave rectified and a linear envelope obtained using the Root Mean Square (RMS) approach with a window size of 300 ms. Data was normalised to the peak muscle activity value over each attempt (\%MAX).

\section{Statistical analysis}

Descriptive statistics were reported as means and standard deviations (mean \pm Std. Deviation). The normality of the data was analyzed using the Shapiro-Wilk test. Independent T-test was used to detect significant differences and compare the mean of each variable between single-leg squat and balance tests. The statistical analysis 
was performed using IBM SPSS software Statistics V21 SPSS Inc., USA) with significance level defined as $p<0.05$ for each test.

\section{Results}

Figure 1 and figure 2 show the single-leg test EMG with and without using of Kinesio tape for the results of the single-leg squat test with (LS_KT) and without (LS), using Kinesio tape of the lower limb selected muscles. Figure 3 and figure 4 show the balance test EMG with and without using of Kinesio tape for the results of the balance test with (Balance_KT) and without (Balance), using Kinesio tape of the lower limb selected muscles.

Figure 1 shows VL, RF, and VM; (A) EMG raw data, (B) EMG rectified data, (C) EMG RMS, and (D) Box-and-whisker plots are presented for normalised EMG (\%MAX) of the $\mathrm{VL}, \mathrm{RF}$, and VM. These values in the single-leg test without $\mathrm{KT}$ were $43.03 \pm 3.95$, $42.22 \pm 9.36,39.99 \pm 3.47$, for $V L, R F$, and VM, respectively. And the results of the singleleg squat test with (KT) values were $42.84 \pm 6.77,40.89 \pm 10.02,42.96 \pm 6.65$, for $V L$, $R F$, and VM, respectively. The results indicated non-significant differences between using $\mathrm{KT}$ or not during the single-leg test in VL, RF, and VM muscles. 
(A)

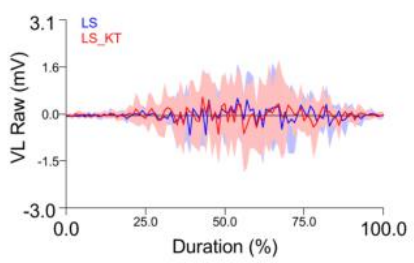

(B)

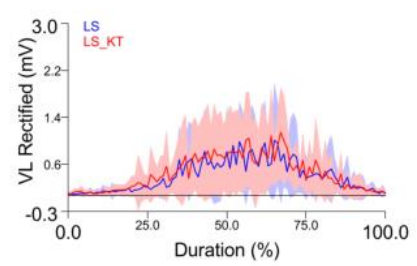

(C)

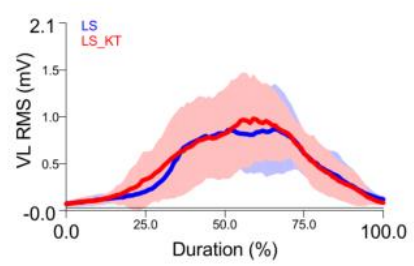

(D)

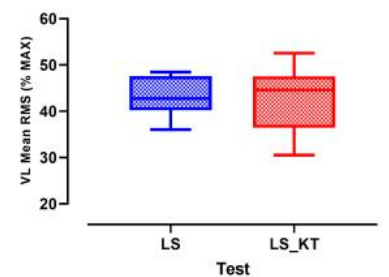

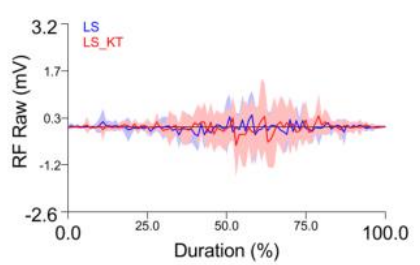
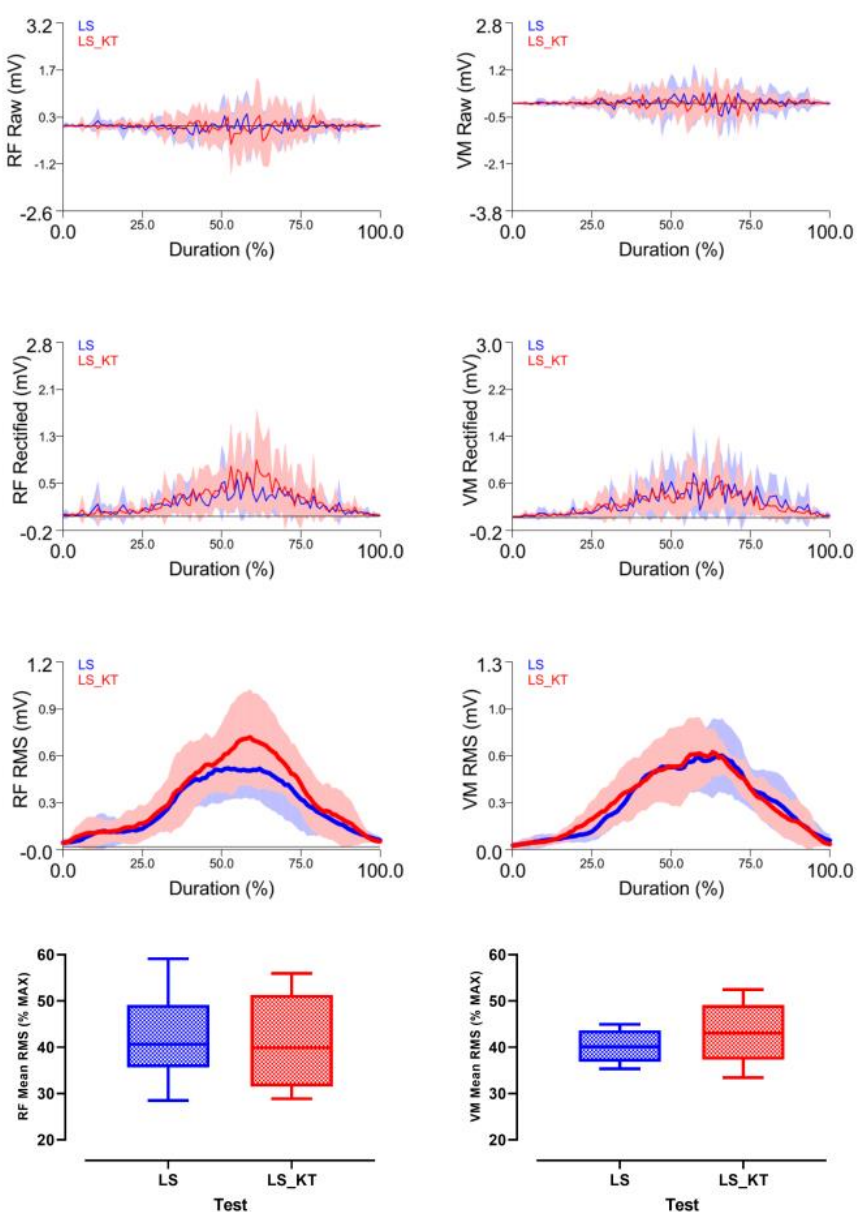

Figure 1. Single-leg test $E M G$ with and without using of Kinesio tape for the VL, RF, and VM; (A) EMG raw data, (B) EMG rectified data, (C) EMG RMS, and (D) Box-and-whisker plots are presented for normalised EMG (\%MAX) of the VL, RF, and VM during the single-leg squat test.

Figure 2 shows BF, ST, L_GAST, and M_GAST; (A) EMG raw data, (B) EMG rectified data, (C)EMG RMS, and (D) Box-and-whisker plots are presented for normalised EMG (\%MAX) of the BF, ST, L_GAST, and M_GAST. These values in the single-leg test without KT were $39.35 \pm 9.64,51.15 \pm 7.41,47.13 \pm 10.50,45.63 \pm 7.41$, for BF, ST, L_GAST, and M_GAST, respectively. And values in the single-leg test with (KT) values were $38.22 \pm 6.38,51.35 \pm 7.99,51.78 \pm 9.68,47.52 \pm 6.02$, for BF, ST, L_GAST, and M_GAST, respectively. The results indicated to a non- BF, ST, L_GAST, and M_GAST muscles. 
(A)

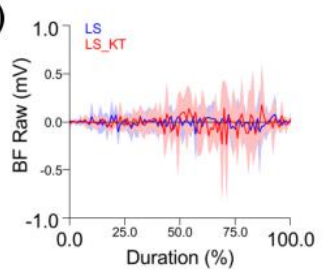

(B)

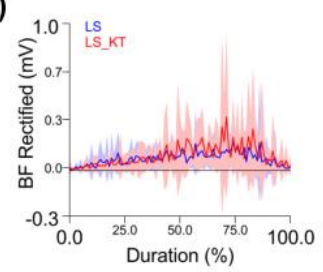

(C)

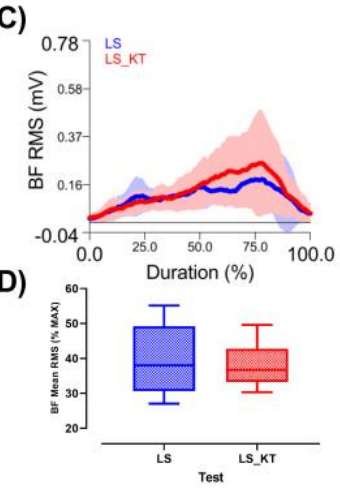

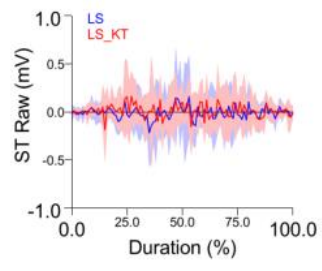
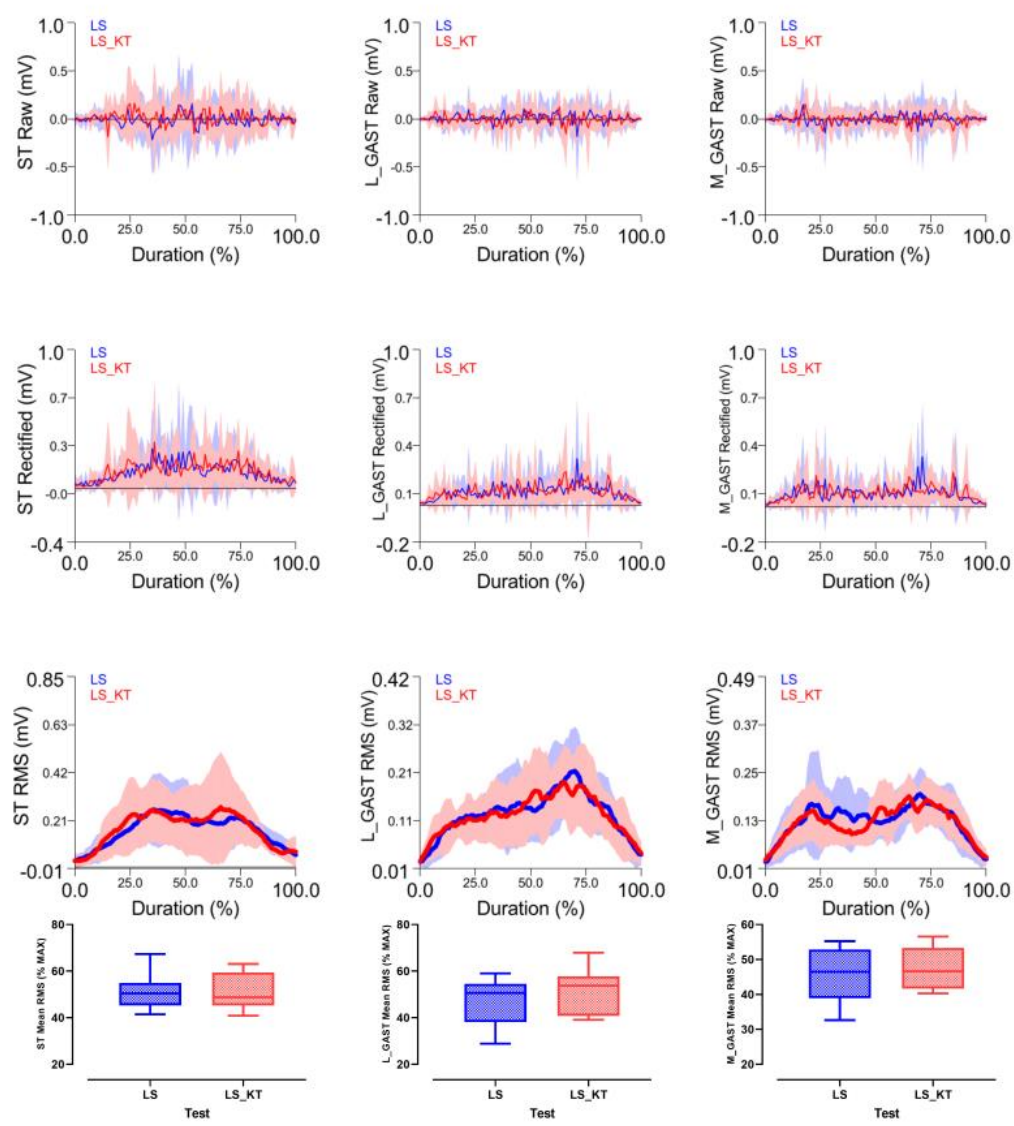

Figure 2. Single-leg test $E M G$ with and without using of Kinesio tape for the $B F, S T, L_{-} G A S T$, and M_GAST; (A) EMG raw data, (B) EMG rectified data, (C)EMG RMS, and (D) Box-andwhisker plots are presented for normalised EMG (\%MAX) of the BF, ST, L_GAST, and M_GAST during the single-leg squat test.

Figure 3 and figure 4 show the balance test EMG with and without using of Kinesio tape for the results of balance test with (Balance_KT) and without (Balance), using Kinesio tape of the lower limb selected muscles.

Figure 3 shows VL, RF, and VM; (A) EMG raw data, (B) EMG rectified data, (C) EMG RMS, and (D) Box-and-whisker plots are presented for normalised EMG (\%MAX) of the $\mathrm{VL}, \mathrm{RF}$, and VM. These values in balance test without $\mathrm{KT}$ were $47.13 \pm 5.19,43.34 \pm$ $10.61,46.94 \pm 6.48$, for $\mathrm{VL}, \mathrm{RF}$, and $\mathrm{VM}$, respectively. And the results of the balance test with (KT) values were $40.44 \pm 5.07,43.20 \pm 11.68,45.90 \pm 5.96$, for $V L, R F$, and VM, respectively. The results indicated to a significant difference between using KT or not during balance test in VL muscle $(p<0.05)$, and non-significant differences between using $\mathrm{KT}$ or not during balance test in RF, and VM muscles. 
(A)

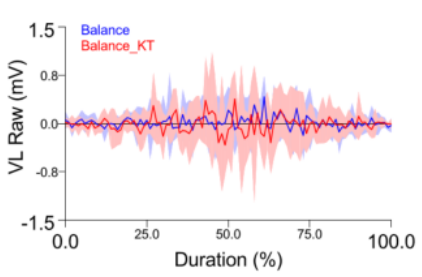

(B)

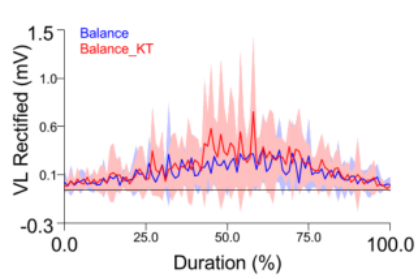

(C)

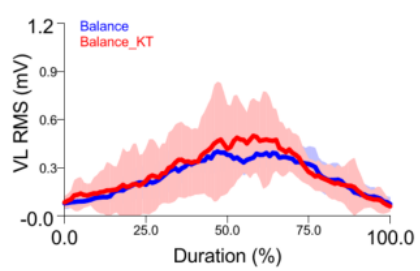

(D)

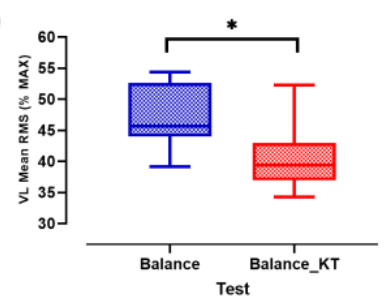

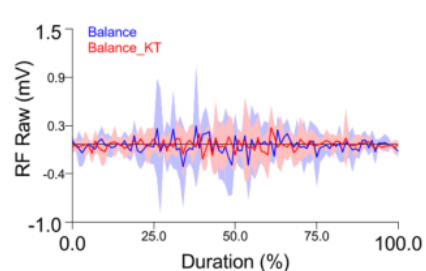
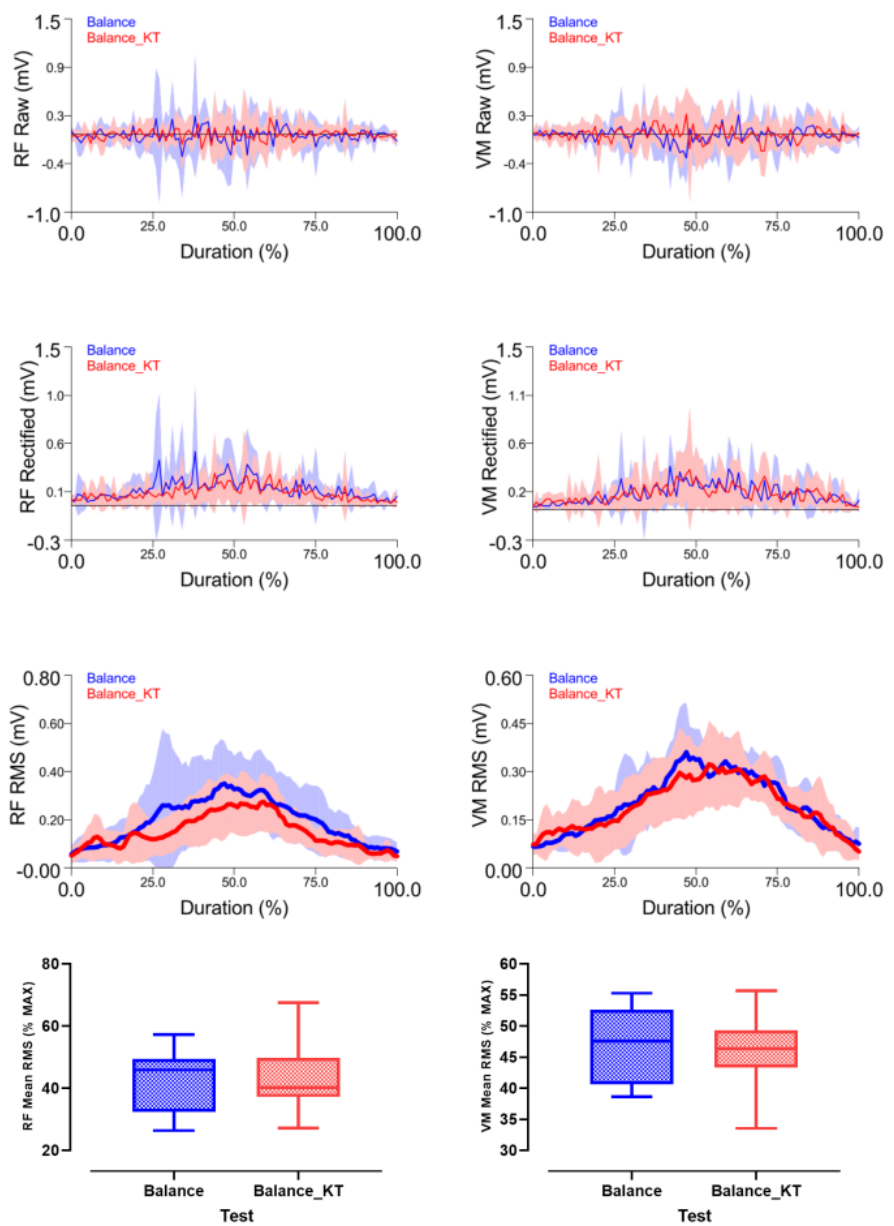

Figure 3. Balance test EMG with and without using of Kinesio tape for the VL, RF, and VM; (A) EMG raw data, (B) EMG rectified data, (C)EMG RMS, and (D) Box-and-whisker plots are presented for normalised EMG (\%MAX) of the VL, RF, and VM during the Balance test.

Figure 4 shows BF, ST, L_GAST, and M_GAST; (A) EMG raw data, (B) EMG rectified data, (C)EMG RMS, and (D) Box-and-whisker plots are presented for normalised EMG (\%MAX) of the BF, ST, L_GAST, and M_GAST. These values in balance test without KT were $44.93 \pm 11.04,48.95 \pm 13.41,43.68 \pm 13.09,43.99 \pm 10.60$, for BF, ST, L_GAST, and M_GAST, respectively. And in balance test with (KT) values were $39.90 \pm 14.60$, $43.00 \pm 12.95,37.41 \pm 16.13,40.67 \pm 13.46$, for BF, ST, L_GAST, and M_GAST, respectively. The results indicated non-significant differences between using KT or not during the balance test in BF, ST, L_GAST, and M_GAST muscles. 
(A)

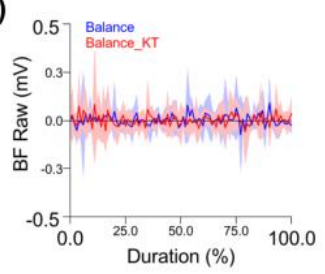

(B)

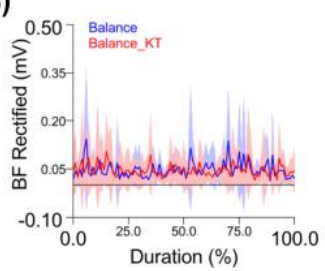

(C)

(D)
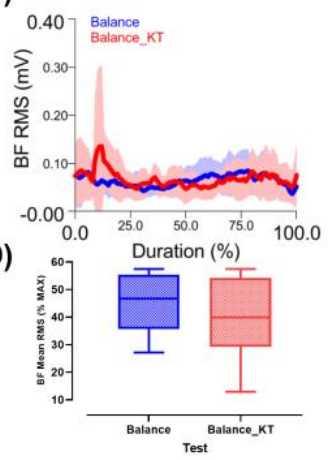
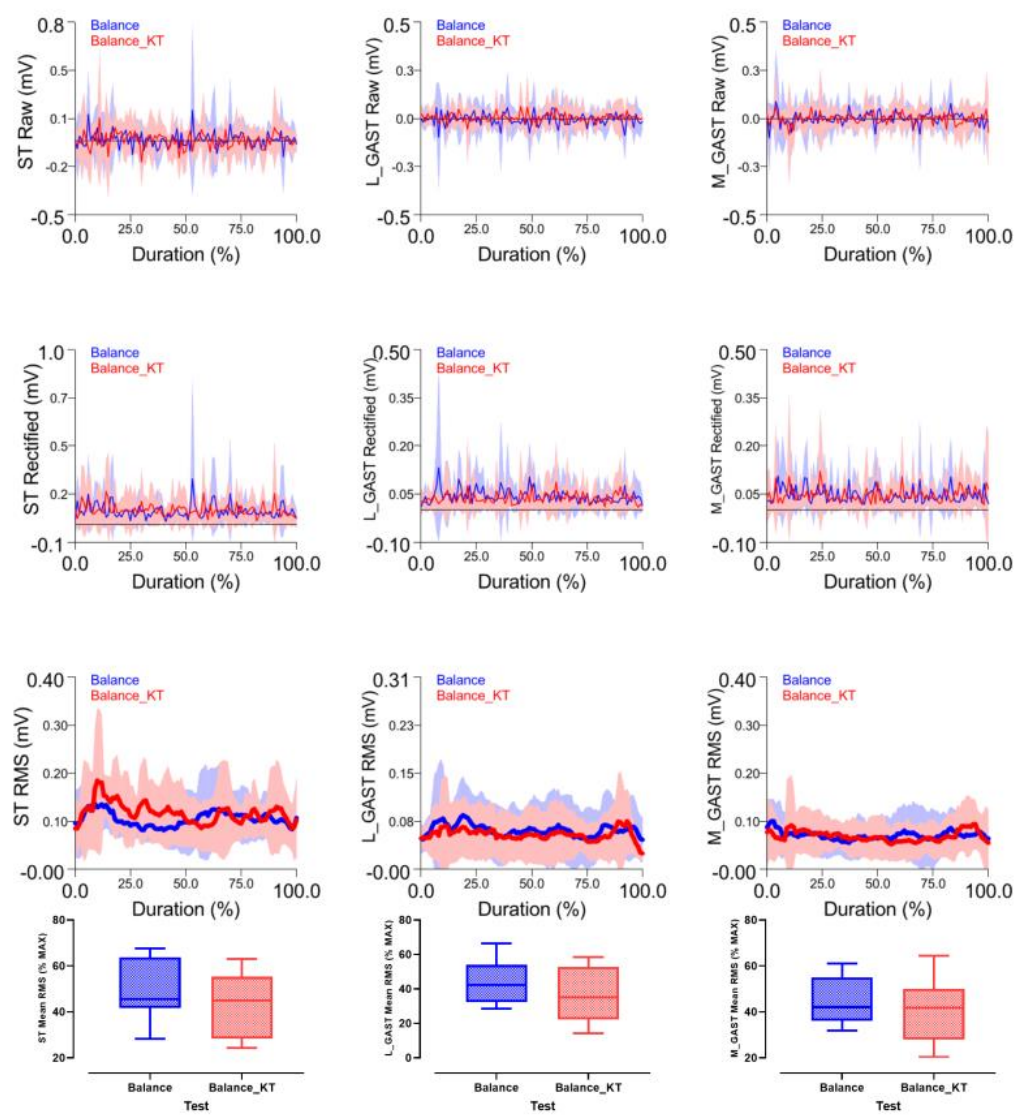
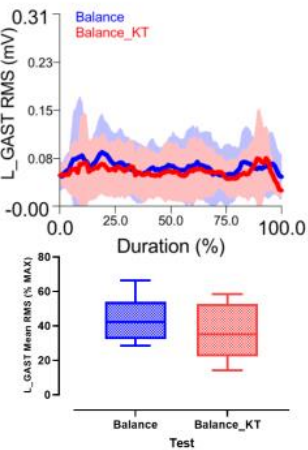
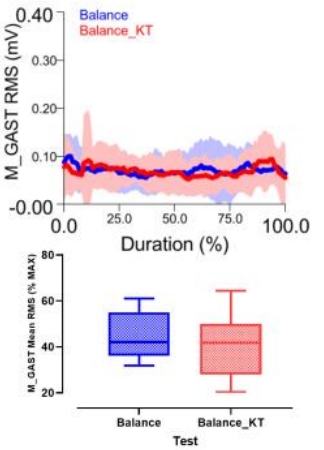

Figure 4. Balance test EMG with and without using of Kinesio tape for the $B F, S T, L \_G A S T$, and M_GAST; (A) EMG raw data, (B) EMG rectified data, (C)EMG RMS, and (D) Box-andwhisker plots are presented for normalised EMG (\%MAX) of the BF, ST, L_GAST, and M_GAST during the Balance test.

\section{Discussion}

We aimed to identify the effect of using Kinesio tape on the neuromuscular activity of lower limb muscles (VL, RF, VM, BF, ST, L_GAST, and M_GAST), after the rehabilitation program of the knee joint, by comparing EMG signal amplitudes between without taping and with KT during single-leg squat and balance tests.

However, our study has some limitations. First of all, we did not include the biomechanical data such as knee angular displacement and lower limb moments which can provide more information about the effects on the knee joint. Another limitation was that the KT applied and EMG test after a short time. Also, randomization generated similar groups in most variables, the duration of symptoms among the two tests were different. Nevertheless, all 
participants were in the $A C L$ reconstruction phase. These limitations should be taken in consideration in future work.

In the present study, no changes were observed in the amplitude of the EMG signal for thigh and calf muscles during a single-leg squat test (Figures 1, 2). Kinesio taping can be applied to muscles to relieve pain, but several studies indicated a nonsignificant effect of KT on muscle activity. (Pires et al., 2019) (Chang et al., 2015). Although KT is widely used in clinical practice and provides positive effects for decreasing the swelling (Balki, Goktas, \& Oztemur, 2016), the current evidence does not support the use of KT (Parreira Pdo, Costa Lda, Hespanhol, Lopes, \& Costa, 2014). These results may due to various reasons such as the duration of applied KT was short, failed to apply sufficient tension during the KT application around the knee, processing EMG signals for the test as one phase, and maybe the KT technique.

Despite these results, Kinesio taping is in widespread clinical use and still has a favourable property and is composed of waterproof and facilitate easy use. Kinesio tape can increase local blood or lymphatic circulation and prevent reactions because it is manufacturing by elastic material similar to the skin and soft tissue (Chang et al., 2015). However, we found no changes in the lower limb muscles (VL, RF, VM, BF, ST, L_GAST, and M_GAST), by analyzing the EMG after KT application during the single-leg squat test.

The balance test was used for examining the effects of KT on the lower limb selected muscles. The present study showed no significant difference in muscle activity between using KT or not during the balance test for all muscles except VL muscle (Figures 3,4 ). The VL muscle activity decreases when using KT during balance test may due to trying the participant in keeping balance and weight-bearing.

However, our study indicated a lack of effects of KT of lower limb selected muscles during single-leg squat and balance tests. According to that applying tape to the selected muscles shortly before the activity of tests does not promote a response in EMG activity, requiring a longer period of the applied KT, to increase the bioelectric performance of the muscle (Melo, Macedo, Borges, \& Brasileiro, 2018).

Finally, we conclude that Kinesio tape doesn't have an adequate effect on the lower limb muscles, but it acts as a catalyst for muscles and leads to a person's feeling of psychological wellbeing and reassurance. 


\section{Conclusion}

The results of the present study showed that applying KT around the knee joint after rehabilitation program does not promote immediate changes or effects on the lower limb muscle activities of (VL, RF, VM, BF, ST, L_GAST, and M_GAST) during the single-leg squat test. And also during balance test except in VL muscle activity.

\section{References}

Aghapour, E., Kamali, F., \& Sinaei, E. (2017). Effects of Kinesio Taping((R)) on knee function and pain in athletes with patellofemoral pain syndrome. J Bodyw Mov Ther, 21(4), 835-839. doi:10.1016/j.jbmt.2017.01.012

Balki, S., Goktas, H. E., \& Oztemur, Z. (2016). Kinesio taping as a treatment method in the acute phase of ACL reconstruction: A double-blind, placebo-controlled study. Acta Orthop Traumatol Turc, 50(6), 628-634. doi:10.1016/j.aott.2016.03.005

Castrogiovanni, P., Di Giunta, A., Guglielmino, C., Roggio, F., Romeo, D., Fidone, F., . . . Musumeci, G. (2016). The Effects of Exercise and Kinesio Tape on Physical Limitations in Patients with Knee Osteoarthritis. Journal of Functional Morphology and Kinesiology, 1(4), 355-368. doi:10.3390/jfmk1040355

Chang, W. D., Chen, F. C., Lee, C. L., Lin, H. Y., \& Lai, P. T. (2015). Effects of Kinesio Taping versus McConnell Taping for Patellofemoral Pain Syndrome: A Systematic Review and Meta-Analysis. Evid Based Complement Alternat Med, 2015, 471208. doi:10.1155/2015/471208

Doğan, E., Yilmaz, A. K., Özdal, M., Mayda, M. H., Yilmaz, Ç., \& Ermiş, E. (2019). Acute effects of reverse Kinesio Taping on knee muscle strength, fatigue index and $H / Q$ ratio in healthy subjects. Isokinetics and Exercise Science, 27, 135-141. doi:10.3233/IES183223

Fu, T. C., Wong, A. M., Pei, Y. C., Wu, K. P., Chou, S. W., \& Lin, Y. C. (2008). Effect of Kinesio taping on muscle strength in athletes-a pilot study. J Sci Med Sport, 11(2), 198-201. doi:10.1016/j.jsams.2007.02.011

Hall, M. P., Paik, R. S., Ware, A. J., Mohr, K. J., \& Limpisvasti, O. (2015). Neuromuscular Evaluation With Single-Leg Squat Test at 6 Months After Anterior Cruciate Ligament Reconstruction. Orthop J Sports Med, 3(3), 2325967115575900. doi:10.1177/2325967115575900

Hebert-Losier, K., Yin, N. S., Beaven, C. M., Tee, C. C. L., \& Richards, J. (2019). Physiological, kinematic, and electromyographic responses to kinesiology-type patella tape in elite cyclists. J Electromyogr Kinesiol, 44, 36-45. doi:10.1016/j.jelekin.2018.11.009

Huang, C. Y., Hsieh, T. H., Lu, S. C., \& Su, F. C. (2011). Effect of the Kinesio tape to muscle activity and vertical jump performance in healthy inactive people. Biomed Eng Online, 10, 70. doi:10.1186/1475-925X-10-70

Juchler, I., Blasimann, A., Baur, H., \& Radlinger, L. (2016). The effect of kinesio tape on neuromuscular activity of peroneus longus. Physiother Theory Pract, 1-6. doi:10.3109/09593985.2015.1110737

Kocyigit, F., Turkmen, M. B., Acar, M., Guldane, N., Kose, T., Kuyucu, E., \& Erdil, M. (2015). Kinesio taping or sham taping in knee osteoarthritis? A randomized, double-blind, sham-controlled trial. Complement Ther Clin Pract, 21(4), 262-267.

doi:10.1016/j.ctcp.2015.10.001 
Melo, S. A., Macedo, L. B., Borges, D. T., \& Brasileiro, J. S. (2018). Effects of kinesio taping on neuromuscular performance and pain of individuals affected by patellofemoral pain: A randomized controlled trial. Physiother Theory Pract, 36(6), 709-719. doi:10.1080/09593985.2018.1492657

Nwe, A. A., Tun, M. T., Aung, S. T., \& Myaing, K. T. (2019). Effectiveness of Kinesio taping in the management of knee osteoarthritis. Journal of Advances in Medicine and Medical Research, 29(2), 1-10.

Parreira Pdo, C., Costa Lda, C., Hespanhol, L. C., Jr., Lopes, A. D., \& Costa, L. O. (2014). Current evidence does not support the use of Kinesio Taping in clinical practice: a systematic review. J Physiother, 60(1), 31-39. doi:10.1016/j.jphys.2013.12.008

Pires, L. G., Padula, R. S., Junior, M., Santos, I., Almeida, M. O., Tomazoni, S. S., . . Costa, L. O. P. (2019). Can Kinesio Taping(R) influence the electromyographic signal intensity of trunk extensor muscles in patients with chronic low back pain? A randomized controlled trial. Braz J Phys Ther. doi:10.1016/j.bjpt.2019.12.001

Poon, K. Y., Li, S. M., Roper, M. G., Wong, M. K., Wong, O., \& Cheung, R. T. (2015). Kinesiology tape does not facilitate muscle performance: A deceptive controlled trial. Man Ther, 20(1), 130-133. doi:10.1016/j.math.2014.07.013

Serrão, J. C., Mezêncio, B., Claudino, J. G., Soncin, R., Miyashiro, P. L. S., Sousa, E. P., . . medicine. (2016). Effect of 3 different applications of Kinesio Taping Denko ${ }^{\circledR}$ on electromyographic activity: inhibition or facilitation of the quadriceps of males during squat exercise. 15(3), 403.

Wageck, B., Nunes, G. S., Bohlen, N. B., Santos, G. M., \& de Noronha, M. (2016). Kinesio Taping does not improve the symptoms or function of older people with knee osteoarthritis: a randomised trial. J Physiother, 62(3), 153-158. doi:10.1016/j.jphys.2016.05.012

Wong, O. M., Cheung, R. T., \& Li, R. C. (2012). Isokinetic knee function in healthy subjects with and without Kinesio taping. Phys Ther Sport, 13(4), 255-258. doi:10.1016/j.ptsp.2012.01.004 